\title{
INTEGRAL observations of recurrent fast X-ray transient sources
}

\author{
V. Sguera ${ }^{1}$, E. J. Barlow ${ }^{1}$, A. J. Bird ${ }^{1}$, D. J. Clark ${ }^{1}$, A. J. Dean ${ }^{1}$, A. B. Hill ${ }^{1}$, L. Moran ${ }^{1}$, \\ S. E. Shaw ${ }^{1,2}$, D. R. Willis ${ }^{1}$, A. Bazzano ${ }^{3}$, P. Ubertini ${ }^{3}$, and A. Malizia ${ }^{4}$
}

\author{
1 School of Physics and Astronomy, University of Southampton, Highfield, SO17 1BJ, UK \\ e-mail: sguera@astro.soton.ac.uk \\ 2 INTEGRAL Science Data Center, 1290 Versoix, Switzerland \\ 3 IASF/CNR, via Fosso del Cavaliere 100, 00133 Roma, Italy \\ 4 IASF/CNR, via Piero Gobetti 101, 40129 Bologna, Italy
}

Received 21 March 2005 / Accepted 17 August 2005

\section{ABSTRACT}

Fast X-ray Transients (FXTs) are believed to be non-recurrent bright X-ray sources lasting less than a day and occuring at serendipitous positions, they can best be detected and discovered by instruments having a sufficiently wide field of view and high sensitivity. The IBIS/ISGRI instrument onboard INTEGRAL is particularly suited to detect new or already known fast X-ray transient sources. We report on IBIS/ISGRI detection of newly discovered outbursts of three fast transient sources located at low Galactic latitude: SAX J1818.6-1703; IGR J16479-4514; IGR J17391-302/XTE J1739-302. The reported results confirm and strengthen the very fast transient nature of these sources, given that all their newly detected outbursts have a duration less than $\sim 3 \mathrm{~h}$. Additionally, they provide the first evidence for a possible recurrent fast transient behaviour as all three sources were detected in outburst by ISGRI more than once during the last 2 years.

Key words. X-rays: bursts - X-rays: individuals: IGR J16479-4514 - X-rays: individuals: SAX J1818.6-1703 - X-rays: general X-rays: individuals: XTE J1739-302/IGR J17391-302 - X-rays: binaries - gamma rays: observations

\section{Introduction}

The X-ray and $\gamma$-ray sky is characterized by marked variability with many sources suddenly appearing and then disappearing again. Amongst the many kinds of variable sources, X-ray transients have been observed since the first X-ray observations. According to their duration, they can be mainly divided into two different classes: long duration and short duration X-ray transients. The former can be visible for weeks or even months and are mainly related to Be-NS binaries systems (Be X-ray transients) or soft X-ray transients (a sub class of low mass $\mathrm{X}$-ray binaries). The latter last from hours to a few days and include, among others, a subclass of sources called Fast X-ray Transients (FXTs) which last from a very few hours to less than a day. Recently, the term X-ray Flash (XRF) has been coined for a new subclass of FXTs lasting less than $1000 \mathrm{~s}$ (Heise et al. 2001), these are thought to be related to gamma ray bursts since the two classes share several observational properties.

Although FXTs are among the most extreme examples of variability in the X-ray sky, not too much is known about them, mainly because of the lack of counterparts at other wavelengths. They occur at unpredictable locations and times and it is very difficult to detect and observe them using traditional narrow field X-ray instruments. On the contrary, detectors having a sufficiently wide field of view are particularly suited to detect fast X-ray transient sources. The larger the field of view of an instrument, the greater the chance of serendipitously detecting a short duration random event, such as a FXT.

To date, only a few hundred FXTs have been observed by such wide field X-ray instruments, from HEAO-1 to BeppoSAX, although their all-sky rate is believed to be of the order of thousands per year (Vadim et al. 2003). Normally, FXTs are non-recurrent bright X-ray sources with no detectable quiescence emission; this implies high peak-to-quiescence flux ratios $\left(10^{2}-10^{3}\right)$ (Ambruster et al. 1983). Less than half of all reported FXTs have been optically identified with stellar flares originating in active galactic coronal sources as RS CVn binaries and dMe-dKe flare stars (Heise et al. 1975; Garcia et al. 1980; McHardy et al. 1982), Algol-type binaries (Schnopper et al. 1976; Favata 1998), W UMa systems and young T Tauri stars. However, FXTs are a heterogeneous collection of objects showing large diversity of observational characteristics (light curves and spectra) which seem to suggest that these events are caused by more than one physical mechanism. In fact many unidentified FXTs do not appear to be coronal events, other progenitors have been suggested such as: dwarf novae (Stern et al. 1981), ordinary and anomalous type I X-ray bursts (Hoffman et al. 1978; Lewis \& Joss 1981), BL Lac objects (Catanese et al. 1997; Maraschi et al. 1999). 
The IBIS/ISGRI detector (Ubertini et al. 2003; Lebrun et al. 2003) on board the INTEGRAL satellite (Winkler et al. 2003) is particularly suited to detect new or already known fast X-ray transient sources. Firstly, INTEGRAL provides a sensitivity higher than any earlier monitoring instruments and high resolution images with a large field of view fully coded $\left(9^{\circ} \times 9^{\circ}\right)$ as well as partially coded (full width at zero response, $30^{\circ} \times 30^{\circ}$ ). Furthermore, the INTEGRAL mission dedicates most of its Core Program to deep exposures of the central Galactic region and scans of the Galactic plane. To date, many new sources have been detected with IBIS/ISGRI, especially toward the inner region of the Galaxy. The emerging picture is that they are High Mass X-ray Binaries (HMXBs), mainly Be/X-ray binaries (Lutovinov et al. 2004). However, there are reasons to think that not all the new ISGRI sources are HMXB, especially those showing a very quick transient nature with activity period less than a day.

Previous to this work no recurring FXTs had been identified, in this paper we report on INTEGRAL observations of three fast X-ray transient sources (SAX J1818.6-1703, IGR J16479-4514 and IGR J17391-302/XTE J1739-302) located at low Galactic latitude and detected by ISGRI more than once during the last 2 years. They are characterized by extremely short outbursts (duration of few hours). We present results on several newly detected outbursts, not previously reported in the literature.

\section{INTEGRAL data analysis}

The reduction and analysis of the IBIS/ISGRI data have been performed by using the INTEGRAL Offline Scientific Analysis (OSA) v.4.1 available to the public through the INTEGRAL Science Data Centre ISDC (Courvoisier et al. 2003). INTEGRAL observations are typically divided into short pointings (Science Window, $\mathrm{ScW}$ ) of $\sim 2000$ s duration. An analysis at the ScW level of the deconvolved ISGRI shadowgrams has been performed to search for transient sources detected (with a significance greater than $6 \sigma$ ) in a few consecutive ScWs in the energy band $20-30 \mathrm{keV}$. The $\mathrm{ScW}$ data set consists of all Core Program observations (the Galactic Plane Survey and the Galactic Centre Deep Exposure). This has been followed by a timing analysis of the newly discovered outbursts, using OSA 4.1 to extract light curves and spectra. Since coded mask instruments are characterized by large field of view (FOV), the brightest sources could affect the detection of the other nearby sources, especially of the weaker ones. Bearing this in mind, we have also investigated the variability pattern of very bright sources in the FOV, besides the sources of interest. They have shown a different time variability enabling us to conclude that the light curves obtained for the newly discovered outbursts are reliable and uncontaminated.

The X-Ray Monitor JEM-X (Lund et al. 2003) on board the INTEGRAL satellite makes observations simultaneously with IBIS/ISGRI, providing images in the energy band 3-35 keV with a 13.2 diameter field of view (although noise towards the edge of the FOV limits the usable area for weak sources to the central 10.5). Images from JEM-X were created for all newly discovered outbursts reported in this paper. In most cases, the source was outside or on the edge of the JEM-X field of view. With one exception (see Sect. 5.2), no significant detections were obtained for those inside the FOV.

\section{SAX J1818.6-1703}

\subsection{Archival $X$-ray observations of the source}

SAX J1818.6-1703 is a fast X-ray transient source which currently does not have any obvious optical counterpart such as a galactic coronal source. It was discovered and detected only once with the Wide Field Camera 2 onboard BeppoSAX on 11 March 1998 at RA $=18^{\mathrm{h}} 18^{\mathrm{m}} 39^{\mathrm{s}} \mathrm{Dec}=-17^{\circ} 03^{\prime} 06^{\prime \prime}$ (error radius $3^{\prime}$ ) (in't Zand et al. 1998). The source was seen to turn on at 19:12:00 UTC and peak quickly (20:38:24 UTC) at a level of $100 \mathrm{mCrab}(2-9 \mathrm{keV})$ and $400 \mathrm{mCrab}(9-25 \mathrm{keV})$. During the last $40 \mathrm{~min}$ of the BeppoSAX observation, the source intensity decreased to an undetectable flux level. Although the WFCs were operational up to April 2002, monitoring the Galactic bulge once per week, no more outbursts of SAX J1818.6-1703 detected by BeppoSAX have been reported in the literature. Despite the fact that IBIS/ISGRI performed numerous Core Program observations with the source in the field of view, to date SAX J1818.6-1703 was not detected with INTEGRAL apart from a weak detection reported by Revnivstev et al. (2004) at a level of $5 \mathrm{mCrab}$ in the energy range $18-60 \mathrm{keV}$, which was not reported as a transient event.

\subsection{New results from analysis of IBIS/ISGRI observations}

We report on detection with IBIS/ISGRI of two outbursts of SAX J1818.6-1703, which are the first to be reported since its discovery with BeppoSAX WFCs in 1998. The times of the 2 newly discovered outbursts are listed in Table 1 together with the energy range over which they have been detected and their fluxes at the peak in the $20-30 \mathrm{keV}$ band. We assumed the beginning of the first $\mathrm{ScW}$ during which the source was detected as being the start time of the outburst and similarly the burst stop time to be the end of the last $\mathrm{ScW}$ during which the source was detected.

Outburst No. 1 (Table 1) was detected by ISGRI on 9 October 2003 only in 2 ScWs (which provides an upper limit on its duration of $\sim 1 \mathrm{~h}$ ) from 20 to $60 \mathrm{keV}$, with a peak flux equal to $\simeq 180 \mathrm{mCrab}$ (energy range $20-30 \mathrm{keV}$ ). Figure 1 shows the sequence of consecutive ScWs (20-30 keV) during which it was observed.

Outburst No. 2 (Table 1 ) was detected only $\sim 1.5$ days later than No. 1. It was observed in $6 \mathrm{ScWs}$ (providing an upper limit on its duration of $\sim 3.5 \mathrm{~h}$ ) only in the energy range $20-30 \mathrm{keV}$. The source turned on at 10 October 2003 21:50:45 UTC and subsequently peaked after $\sim 35 \mathrm{~min}$ (10 October 2003 22:25:14 UTC) at a level of $185 \mathrm{mCrab}$. Subsequently the intensity decreased to an undetectable flux level on 11 October 2003 01:00:12 UTC. Figure 2 shows its ISGRI light curve (20-30 keV), while Fig. 3 shows the sequence of consecutive ScWs (20-30 keV) during which it was observed. 
Table 1. Newly discovered outbursts of SAX J1818.6-1703.

\begin{tabular}{|c|c|c|c|c|c|}
\hline No. & Date & Burst start time (UTC) & Burst stop time (UTC) & Energy range & flux at the peak $(20-30 \mathrm{keV})$ \\
\hline 1 & 9 October 2003 & $13: 06: 48$ & $14: 08: 57$ & $20-60 \mathrm{keV}$ & $178 \mathrm{mCrab}$ \\
\hline 2 & 10 October 2003 & $21: 50: 45$ & 01:00:12 & $20-30 \mathrm{keV}$ & $185 \mathrm{mCrab}$ \\
\hline
\end{tabular}

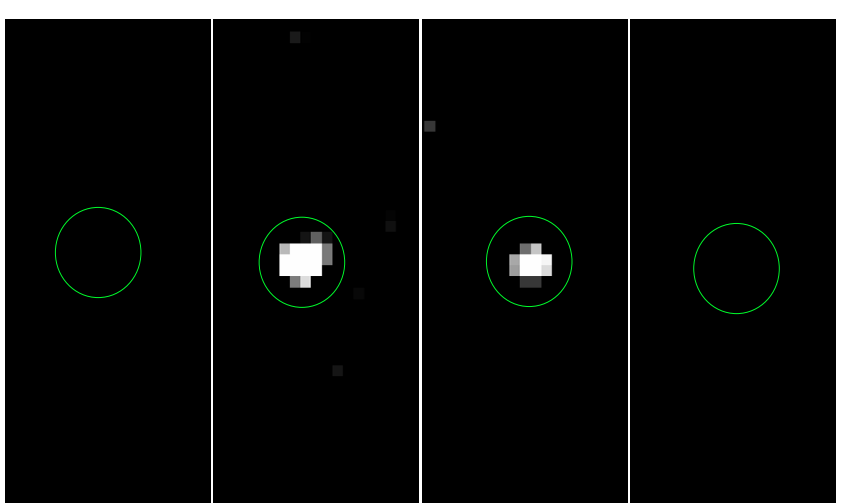

Fig. 1. ISGRI Science Window image sequence $(20-30 \mathrm{keV})$ of a newly discovered outburst (No. 1 in Table 1) of SAX J1818.6-1703 (encircled). The duration of each $\mathrm{ScW}$ is $\sim 2000 \mathrm{~s}$. Significance of the detection, left to right, was $<2 \sigma, 11 \sigma, 8 \sigma,<1 \sigma$.

We can note that these 2 newly discovered outbursts have a very similar peak flux value, both being $\simeq 180 \mathrm{mCrab}$ in the 20-30 keV energy band. Moreover their duration (respectively $\sim 1 \mathrm{~h}$ and $\sim 3 \mathrm{~h}$ ) is quite similar to that of the outburst detected by BeppoSAX WFCs when the source was discovered in 1998 ( $\sim 2 \mathrm{~h})$.

An investigation of the RXTE ASM (All Sky Monitor) data archive provided a light curve (2-12 keV) of SAX J1818.6-1703 from 1996 to 2004 (see Fig. 4) which shows at least 6 more outbursts having a flux greater than $250 \mathrm{mCrab}$ in the energy range $2-12 \mathrm{keV}$ (see numbers in Fig. 4). A check of the RXTE light curve data points showed that there are none at the epoch of the outbursts detected by BeppoSAX WFCs and IBIS/ISGRI. This can be clearly seen in the insets in Fig. 4 which show a zoomed view of the RXTE light curve during the epoch of the BeppoSAX WFCs and IBIS/ISGRI observations, marked by arrows. It can be noted that the outburst detected by BeppoSAX WFCs is very close to one of those detected by RXTE (outburst No. 2 in Fig. 4), the former occurred 17.95 days earlier.

\section{IGR J16479-4514}

\subsection{Archival X-ray observations of the source}

IGR J16479-4514 was discovered with IBIS/ISGRI during observations of the Galactic Center region performed between August 8 and August 92003 (Molkov et al. 2003), the measured fluxes were $12 \mathrm{mCrab}$ and $8 \mathrm{mCrab}$ in the energy bands $18-25 \mathrm{keV}$ and $25-50 \mathrm{keV}$ respectively. During observations performed on August 10, the source showed an increased outburst activity by a factor $\sim 2$ in the energy bands $18-25 \mathrm{keV}$ and $25-50 \mathrm{keV}$, moreover it was also detected in the energy band 50-100 keV with a flux equal to $\sim 17 \mathrm{mCrab}$

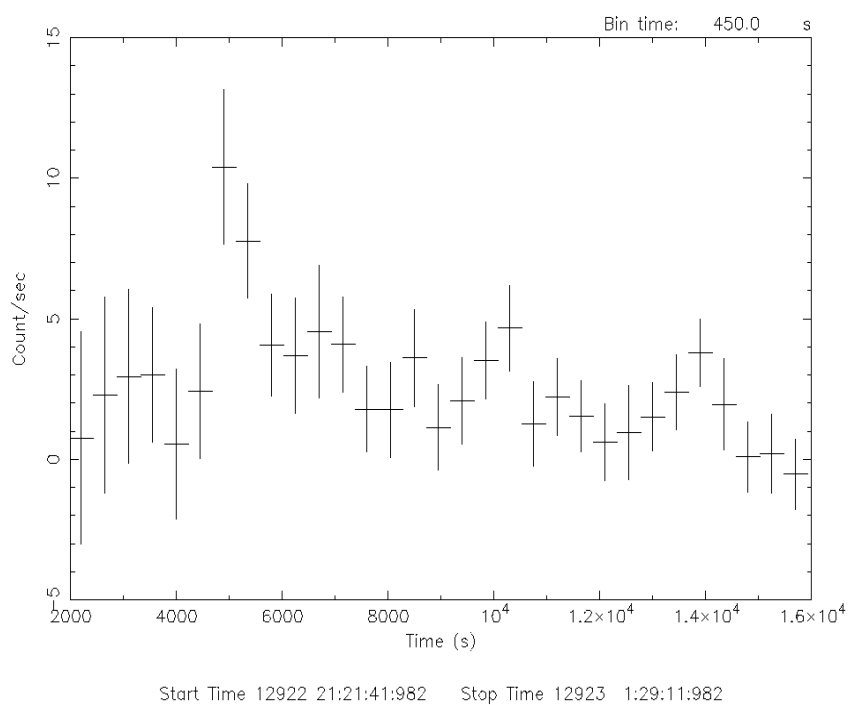

Fig. 2. ISGRI light curve (20-30 keV) of a newly discovered outburst (No. 2 in Table 1) of SAX J1818.6-1703.

(Molkov et al. 2003). To date this is the only outburst of IGR J16479-4514 to be mentioned in the literature. This outburst was reported as an average detection during an observation of the Galactic center field lasting about 3 days (Molkov et al. 2003), and no analysis at the Science Window level was performed on this outburst, hence the fast transient nature of the source was not reported. Recently Lutovinov et al. (2004) published a broad band energy spectrum of IGR J16479-4514 (1-100 keV) described by a simple power law model modified by the cutoff at high energies and the photoabsorption at soft Xrays. The best fit parameters are a photon index of $\Gamma=1.4 \pm 0.8$, an observed absorption of $N_{\mathrm{H}} \sim 1.2 \times 10^{23} \mathrm{~cm}^{-2}$ (exceeding the galactic value along the line of sight) and a high energy cut-off having a value of $E_{\mathrm{c}} \sim 32 \mathrm{keV}$. Based on these spectral characteristics, Lutovinov et al. (2004) argue that IGR J16479-4514 should be a neutron star binary system with high mass companion (HMXB).

The IGR J16479-4514 error circle has a radius of $\sim 3^{\prime}$, no $\mathrm{X}$-ray and radio sources have been found inside this radius using all available catalogues in the HEASARC database. As for the infra-red band, Fig. 5 shows the ISGRI error circle superimposed on the IRAS infra-red field $(12 \mu \mathrm{m})$ as taken from the IRAS Sky Survey Atlas (ISSA) data archive. We note that on the edge of the ISGRI error circle there is a strong IRAS source (IRAS 16441-4506, located 2.9' from the ISGRI position) which is also identified with MSX5C G340.1271-00.0681 and a 2MASS infra-red source (see Table 2 for their fluxes). IRAS and MSX spectral bands (far infra-red) are essentially unaffected by dust or gas absorption, while on the contrary the 2MASS spectral band (near infra-red) is strongly affected by absorption. From Table 2, we can note that 2MASS flux 

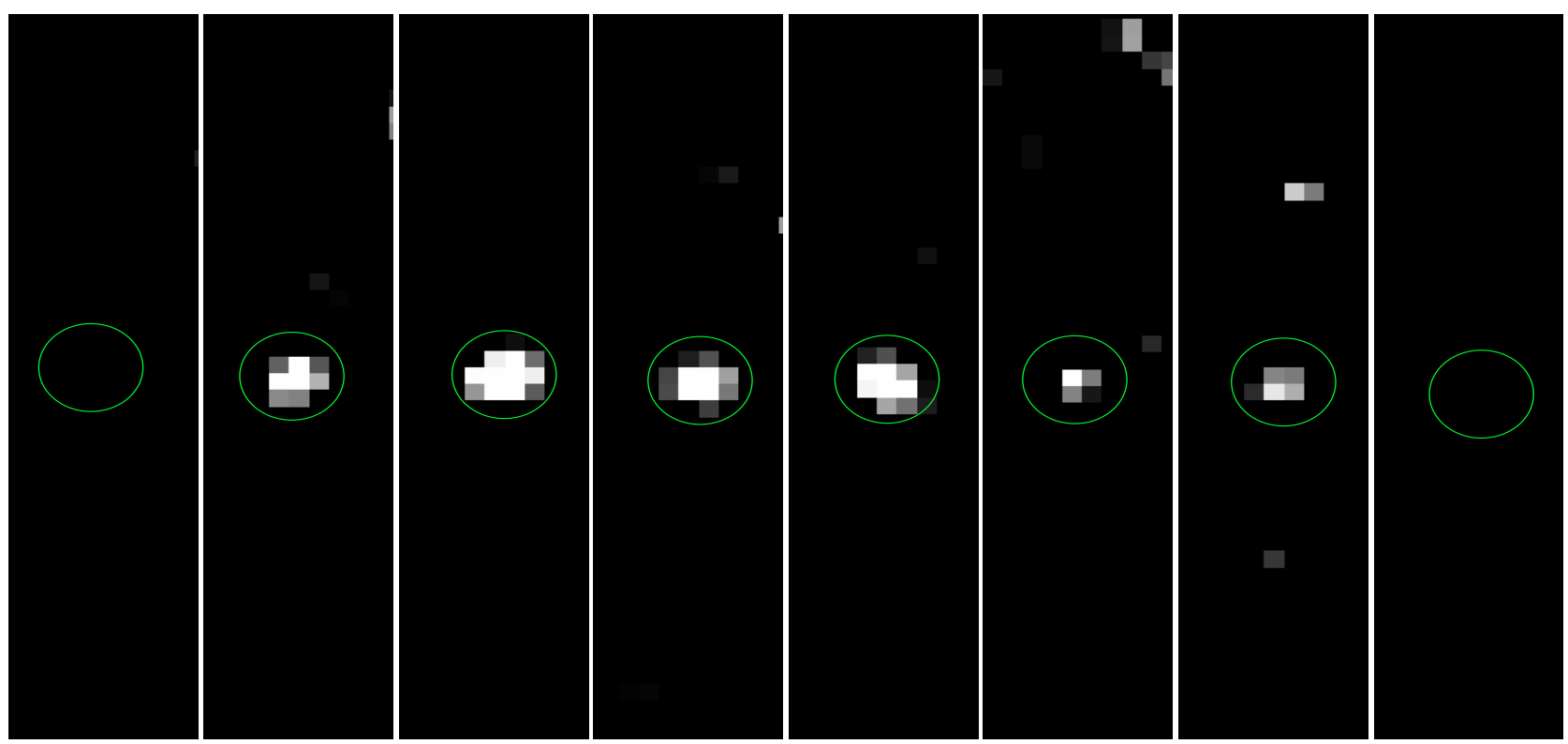

Fig. 3. ISGRI Science Window ( $\mathrm{ScW}$ ) image sequence $(20-30 \mathrm{keV})$ of a newly discovered outburst (No. 2 in Table 1) of SAX J1818.6-1703 (encircled). The duration of each $\mathrm{ScW}$ is $\sim 2000 \mathrm{~s}$. The source was not detected in the first $\mathrm{ScW}$ on the left (significance less than $1 \sigma$ ), then it was detected during the next $6 \mathrm{ScWs}$ with a significance, from left to right, equal to $5 \sigma, 10.5 \sigma, 7.5 \sigma, 7 \sigma, 5 \sigma$ and $4.5 \sigma$, respectively. Finally in the last $\mathrm{ScW}$ the source was not detected (significance less than $2 \sigma$ ).

Table 2. IRAS, MSX and 2MASS infra-red fluxes of the source IRAS 16441-4506.

\begin{tabular}{cc}
\hline \hline $\begin{array}{c}\text { Wavelength } \\
(\mu \mathrm{m})\end{array}$ & $\begin{array}{c}\text { Flux } \\
(\mathrm{Jy})\end{array}$ \\
\hline IRAS 12 & 50.51 \\
IRAS 25 & 44.6 \\
IRAS 60 & 21.86 \\
IRAS 100 & 230.2 \\
MSX 12 & 55.46 \\
MSX 15 & 51.07 \\
MSX 21 & 46.82 \\
2MASS 1.25 & 0.005 \\
2MASS 1.65 & 1.14 \\
2MASS 2.17 & 5.37 \\
\hline
\end{tabular}

measurements are much smaller than those of IRAS and MSX, which suggests that IRAS 16441-4506 could be a strongly absorbed source with a column density possibly exceding the value along the line of sight, which is $\sim 10^{22} \mathrm{~cm}^{-2}$ (Dickey \& Lockman 1990). If IRAS $16441-450$ is the infra-red counterpart of IGR J16479-4514, this is in agreement with the high observed absorption of IGR J16479-4514 $\left(N_{\mathrm{H}} \sim 1.2 \times\right.$ $10^{23} \mathrm{~cm}^{-2}$ ) reported by Lutovinov et al. (2004). Moreover, IRAS 16441-4506 has been classified as an unusual source because its spectrum shows a strange and very strong peak emission near $11 \mu \mathrm{m}$ (Volk et al. 1989). In the IRAS image (Fig. 5), an infra-red ring-like structure is clearly visible. Apart from IRAS 16441-4506, the two other very strong IRAS sources (A and B in Fig. 5) are classified as HII regions (SIMBAD). IGR J16479-4514 seems to be located in an active star formation region, unless this is a projection effect.

\subsection{New results from analysis of IBIS/ISGRI observations}

We report on 4 newly discovered outbursts of IGR J16479-4514, derived from analysis of ISGRI data in revolutions number 47, 55, 63 and 101, respectively. The times of the 4 outbursts are listed in Table 3 together with the energy range over which they have been detected and their fluxes in the 20-30 keV band. We assumed the beginning of the first $\mathrm{ScW}$ during which the source was detected as being the start time of the outburst and similarly the burst stop time to be the end of the last $\mathrm{ScW}$ during which the source was detected. We can note that their duration ranges from $\sim 30 \mathrm{~min}$ to $\sim 3 \mathrm{~h}$, marking the very fast transient nature of IGR J16479-4514, moreover they bracket in time the only outburst so far reported in the literature (8-10 August 2003).

Outburst No. 1 in Table 3 is particularly interesting, being by far the brightest one. Its ISGRI light curve $(20-30 \mathrm{keV})$ is shown in Fig. 6. Initially the source flux is consistent with zero, then suddenly turns on at 13:36:37 UTC, flares up and quickly reaches the peak after $\sim 5 \min (13: 41: 39$ UTC). Then it drops to a very low flux level in $\sim 25 \mathrm{~min}$ and continues to be characterized by a very low flux showing some secondary peaks, as can be seen in Fig. 6. At 16:58:30 UTC the source completely disappears. The count rate at the peak is nearly $50 \mathrm{c} \mathrm{s}^{-1}$ (energy band $20-30 \mathrm{keV}$ ) which is equal to a flux of $850 \mathrm{mCrab}$ or $3.8 \times 10^{-9} \mathrm{erg} \mathrm{cm}^{-2} \mathrm{~s}^{-1}$. Being located in the direction of the Norma region, an active star formation region, we can assume a distance for IGR J16479-4514 of $6 \mathrm{kpc}$, in this case the $20-30 \mathrm{keV}$ luminosity is $1.6 \times 10^{37} \mathrm{erg} \mathrm{s}^{-1}$. Figure 7 shows an expanded view of the outburst light curve binned in $100 \mathrm{~s}$ periods, compared to the $250 \mathrm{~s}$ used in Fig. 6 . There is evidently a fast rise of the flare followed by a slower decay during which 


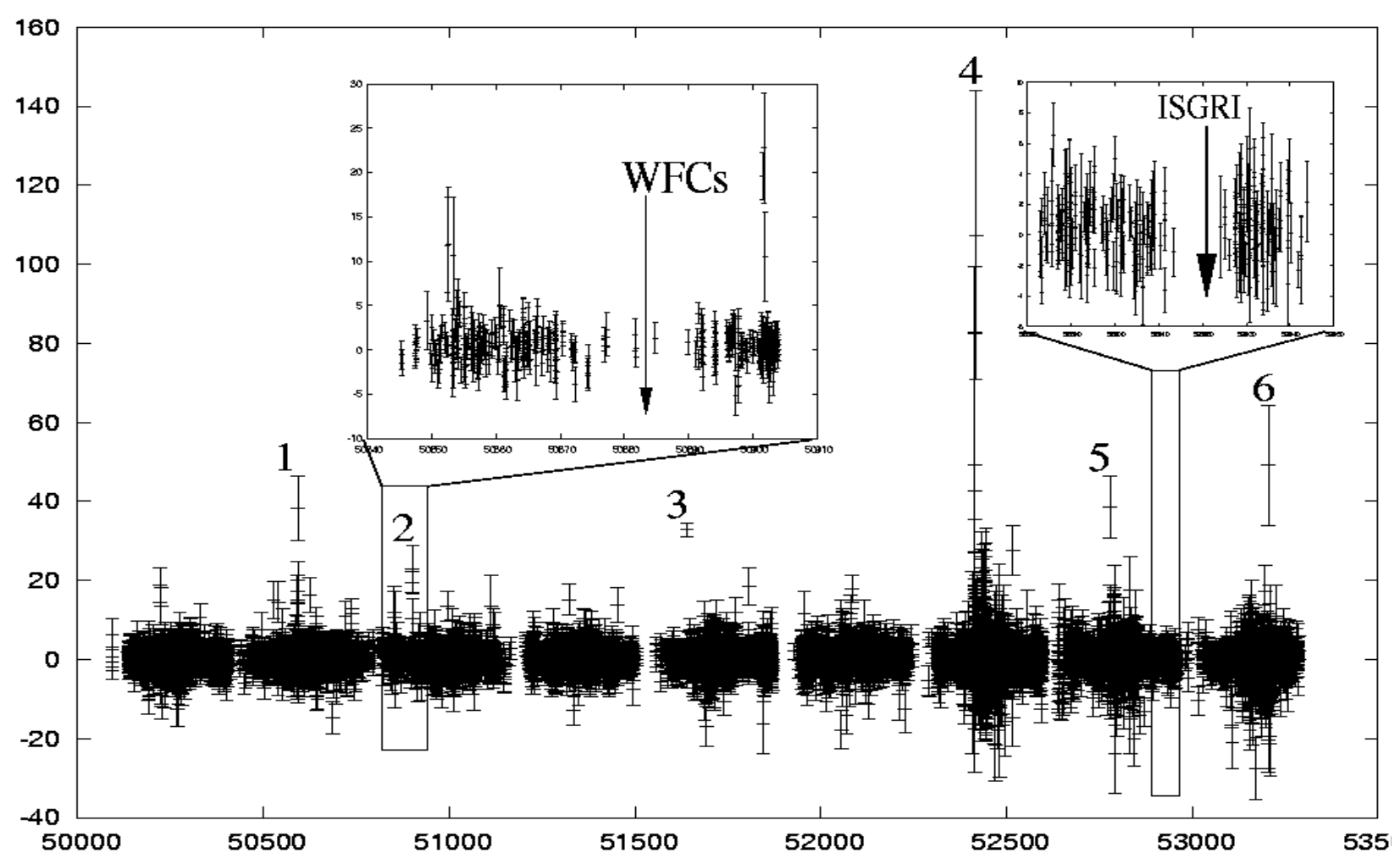

Fig. 4. RXTE ASM dwell by dwell light curve (2-12 keV) of SAX J1818.6-1703 from 1996 to 2004. In the light curve $20 \mathrm{c} \mathrm{s}^{-1}$ is equivalent to a flux of 250 mCrab. Time axis is in MJD. The six outbursts detected by RXTE, with a flux greater than 250 mCrab, are indicated in the light curve by the numbers from 1 to 6 . Zoomed views of the RXTE light curve during the epoch of the BeppoSAX WFCs and IBIS/ISGRI observations (marked by arrows) are shown in the insets, highlighting the lack of RXTE coverage during the outbursts. The two ISGRI outbursts are indicated in the inset on the right by means of only one arrow since they are temporally very close each other. ASM data can be retrieved on the public archive at http://xte.mit.edu/XTE/asmlc/ASM.html

Table 3. Newly discovered outbursts of IGR J16479-4514.

\begin{tabular}{clcccc}
\hline \hline No. & Date & Burst start time (UTC) & Burst stop time (UTC) & Energy range & Flux (20-30 keV) \\
\hline 1 & 5 March 2003 & $13: 36: 37$ & $16: 58: 30$ & $20-60 \mathrm{keV}$ & $850 \mathrm{mCrab}$ \\
2 & 28 March 2003 & $07: 44: 24$ & $09: 18: 49$ & $20-40 \mathrm{keV}$ & $37 \mathrm{mCrab}^{\dagger}$ \\
3 & 21 April 2003 & $08: 51: 34$ & $09: 28: 14$ & $20-60 \mathrm{keV}$ & $160 \mathrm{mCrab}^{\dagger}$ \\
4 & 14 August 2003 & $00: 19: 29$ & $02: 07: 44$ & $20-60 \mathrm{keV}$ & $44 \mathrm{mCrab}^{\dagger}$ \\
\hline
\end{tabular}

Note: $\dagger=$ Average flux $(20-30 \mathrm{keV})$ during the outburst. $\star=$ Flux $(20-30 \mathrm{keV})$ at the peak of the outburst.

there is an indication of a possible secondary peak. We fitted an exponential function $F=F(0) \mathrm{e}^{-t / \tau}$ to the observed flux count rate during the decay in the $20-30 \mathrm{keV}$ energy band. This gives a $\chi_{v}^{2}=0.8$ for 15 d.o.f. and $\tau=15 \pm 9$ min, so it is reasonable to assert an exponential behavior for the decay of the outburst.

As for the remaining outbursts (Nos. 2, 3 and 4 in Table 3), they are shorter than No. 1 having been detected only in few ScWs (3, 1 and 3, respectively) during which their average fluxes (20-30 keV) are respectively $37 \mathrm{mCrab}, 160 \mathrm{mCrab}$ and 44 mCrab. It can be noted that outburst No. 4 in Table 3 was detected a few days later ( $\sim 5$ days) than that reported by Molkov et al. (2003) when IGR J16479-4514 was discovered.

We performed a Lomb-scargle periodogram analysis to search for periodicities using the ISGRI data of the four outbursts listed in Table 3, but no statistically significant periodicities have been found from 0.001 to $500 \mathrm{~Hz}$.
Bearing in mind its spectral characteristics and its apparent location in a star formation region, IGR J16479-4514 could be a HMXB, as previously suggested by Lutovinov et al. (2004). In the light of this interpretation, the 4 newly discovered outburts reported in Table 3 are possibly due to changes in the mass accretion rate onto the compact object (neutron star or black hole). The variations of the mass accretion rate can be related to an eccentric orbit, changes in the companion star or instabilities of the accretion disk. However the durations of the four newly discovered outbursts (ranging from $\sim 30 \mathrm{~min}$ to $\sim 3 \mathrm{~h}$ ) are very unusual for a HMXB given that it makes them significantly shorter than the type II outbursts of Be/NS binaries, which typically last for several weeks or even months.

We can note in Fig. 7 that at first glance the time behavior of outburst No. 1 (very rapid rise lasting $\sim 5 \mathrm{~min}$ followed by a slower exponential decay lasting $\sim 25 \mathrm{~min}$ ) recalls a 


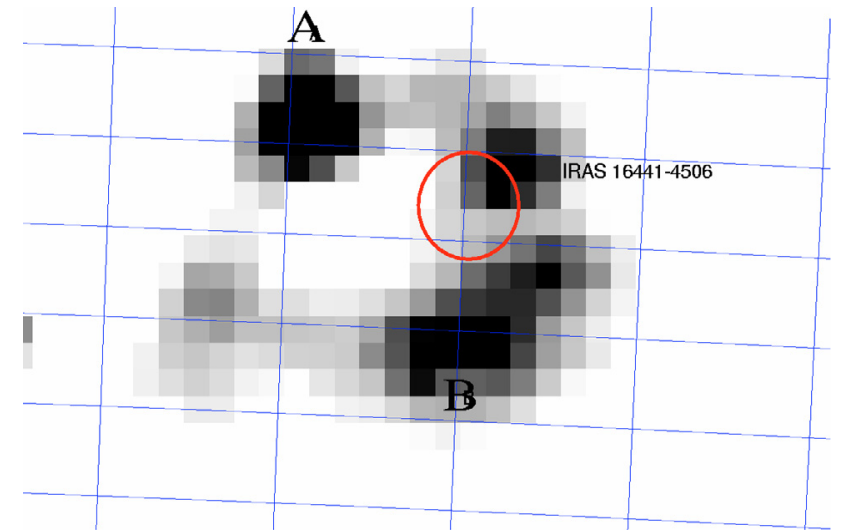

Fig. 5. IRAS infra-red picture $(12 \mu \mathrm{m})$ superimposed on the ISGRI IGR J16479-4514 error circle (3 arcmin radius).

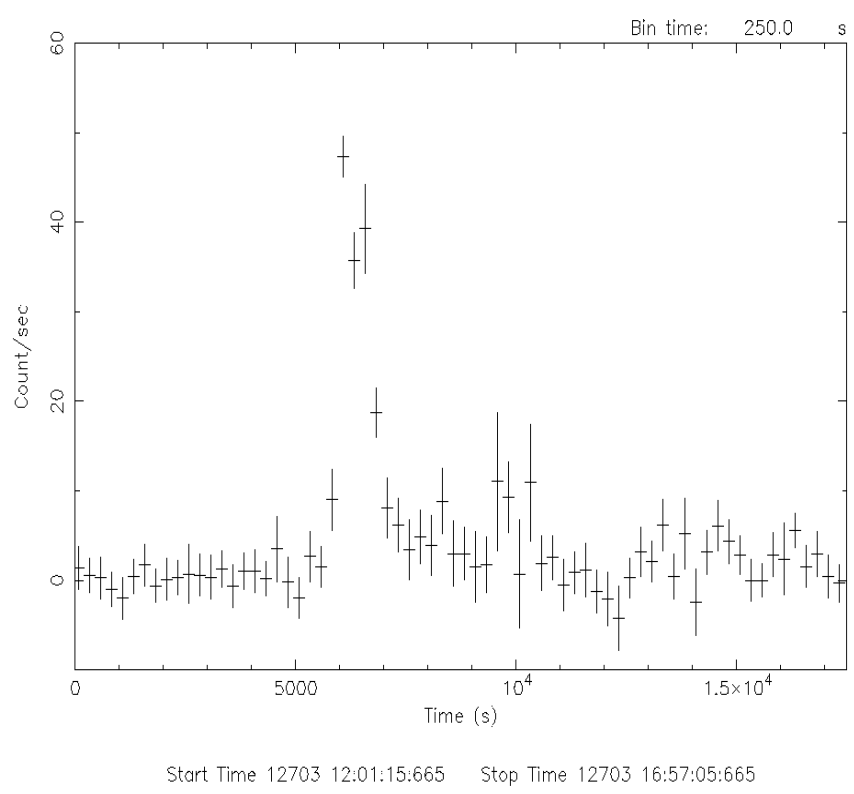

Fig. 6. ISGRI light curve (20-30 keV) of a newly discovered outburst (No. 1 in Table 2) of IGR J16479-4514.

thermonuclear type I X-ray burst. About $40 \%$ of the LMXBs in our Galaxy show type I X-ray bursts, which are explained as energy release by rapid nuclear fusion of material on the surface of a neutron star. Thus a type I X-ray burst is thought to identify the source emitting it unambiguously as a LMXB containing a neutron star (for reviews, see Lewin et al. 1993 and Strohmayer \& Bildsten 2003). Normally they are characterized by rise times lasting from less than a second to $\sim 10 \mathrm{~s}$, followed by a slower exponential decay lasting from $10 \mathrm{~s}$ to minutes. During the decline they also show cooling of the characteristic temperature of the X-ray spectrum which is attributed to cooling of the neutron star surface. The properties of a type I X-ray burst depend, according to theory, on the mass and radius of the neutron star, on the rate with which material is accreted onto the neutron star and on the composition of the accreted material. Some longer type I X-ray bursts have been detected in many other sources. In order of decreasing duration, some examples are: 4 U1708-23, $\simeq 25$ min (Hoffman et al. 1978; Lewin et al. 1984); 4U1724-307, $\geq 10 \mathrm{~min}$

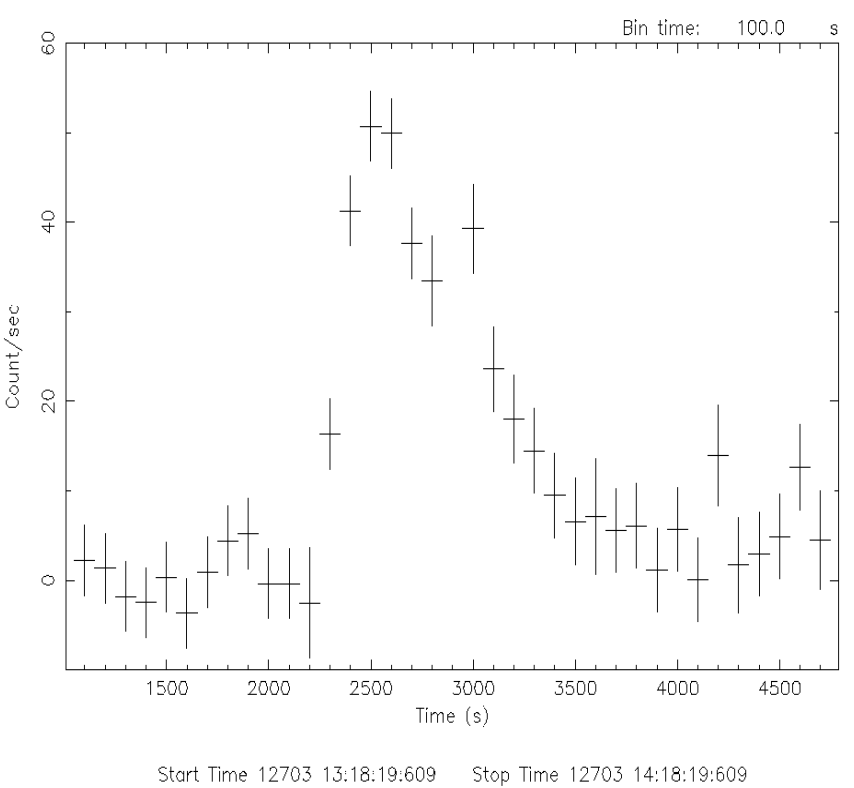

Fig. 7. Expanded view of the light curve (20-30 keV) of the outburst from IGR J16479-4514 showed in Fig. 6.

(Swank et al. 1977); 3A1715-321, $\geq 4.5$ min (Tawara et al. $1984 a, b)$. It is worth noting that even longer type I X-ray bursts exist, the so-called superbursts, lasting for several hours (Cornelisse et al. 2000, 2002; Wijnands 2001; Strohmayer \& Brown 2002; Kuulkers 2001; Kuulkers et al. 2002). Superbursts are more energetic and longer in duration than typical type I Xray bursts, but with similar spectral evolution. This suggests that they result from thermonuclear flashes occuring in fuel layers at much greater depth than typical type I X-ray bursts, thereby accounting for their longer duration. The recurrence times of these events are not well constrained, so far only 7 events have been found, indicating that they are rare.

Typical type I X-ray outburst spectra are well described by a black body emission with temperature of $\sim 2-3 \mathrm{keV}$. The temperature increases during the outburst rise (reflecting the heating of the neutron star surface) and decreases during the decay (due to subsequent cooling). We performed a spectral analysis of the rise of outburst No. 1 as well as of its exponential decay, to find evidence of the cooling of the characteristic temperature of the X-ray spectrum, which would be a clear signature of a type I X-ray burst. To this aim, first we created a user defined Good Time Interval (GTI) file to be used by OSA4.1 to extract the spectrum of IGR J16479-4514 during the rise and the exponential decay. We rebinned the latest response matrix from the original 2048 channels to 64 channels, the channels in the range $14-45 \mathrm{keV}$ were grouped in bins of about $2 \mathrm{keV}$ in size, while the ones in the range $45-100 \mathrm{keV}$ were grouped in bins of about $5 \mathrm{keV}$ size. This was done to have enough statistics in the energy range $20-100 \mathrm{keV}$ to perform the $\chi^{2}$ test in the spectral fit with XSPEC.

As for the 20-60 keV spectrum extracted during the rise, the best fit model is a black body $\left(\chi_{v}^{2}=1.6\right.$, d.o.f. 14) with a temperature equal to $k T=6.8 \pm 0.6 \mathrm{keV}$. Other spectral models, such as thermal bremsstrahlung or Comptonization models, provided instead very poor fits with a $\chi_{v}^{2}$ greater than 1.9. 
The 20-60 keV spectrum extracted during the exponential decay can be fitted by black body model, giving a reasonable description $\left(\chi_{v}^{2}=1.5\right.$, d.o.f. 14$)$, with a temperature equal to $k T=6.4 \pm 0.3 \mathrm{keV}$. However a similarly reasonable fit was also achieved using a thermal bremsstrahlung or Comptonization model (CompST in XSPEC). Clearly, the temperatures of the black body emission during the rise and the exponential decay are comparable within the errors of the measurements so the statistics are not good enough to find evidence of the cooling of the characteristic temperature of the X-ray spectrum, which would be a clear signature of a type I X-ray burst. The black body spectrum is characterized by a temperature higher than typical type I X-ray bursts $(\sim 2-3 \mathrm{keV})$, although black body temperatures in excess of $3 \mathrm{keV}$ were observed for several sources (Lewin et al. 1993). Numerical model calculations of the radiative transfer in neutron star atmospheres show that, although the shape of the spectrum is very nearly Planckian, in some cases the neutron stars are not very good black body emitters during the X-ray burst. The temperature fitted to the spectrum (i.e. the colour temperature $T_{\mathrm{c}}$ ) can be higher than the effective temperature $\left(T_{\text {eff }}\right)$ of the neutron star atmosphere with values of the ratio $T_{\mathrm{c}} / T_{\mathrm{eff}}$ as high as 1.7 (London et al. 1984; Foster et al. 1986; Ebisuzaki \& Nomoto 1986).

On the one hand the energetic and the temporal behavior of the outburst (fast rise followed by a slower exponential decay) recalls a type I X-ray burst, while conversely it was not possible to find evidence of the cooling. Hence a type I X-ray burst as explanation for the outburst of IGR J16479-4514 is inconclusive if only based on the temporal behavior of the outburst. If outburst No. 1 is a type I X-ray burst, this would identify IGR J16479-4514 as a LMXB containing a neutron star. However, this kind of explanation contrasts with the apparent source location in a star formation region of the Norma spiral arm and so with a possible HMXB nature, as was already proposed by Lutovinov et al. (2004), unless the apparent location of IGR J16479-514 in the Norma region is a projection effect. To this aim, we inferred an upper limit to the distance of the source. If it is indeed a type I X-ray burst, it should be Eddington limited with a peak flux less than or equal to the Eddington limit, which for canonical neutron star values is $2 \times 10^{38} \mathrm{erg} \mathrm{s}^{-1}$. By setting the Eddington limit equivalent to the bolometric observed flux we can calculate an upper limit to the distance, bearing in mind that this kind of distance determination to IGR J16479-4514 is only a rough estimate. By doing so an upper limit of $\sim 16 \mathrm{kpc}$ was obtained. This value leaves the possibility that the location of IGR J16479-4514 in a star formation region of the Norma spiral arm is only a projection effect. Unfortunately the value is too high to give any meaningful constraint to the distance and it might be due to the wrong assumption that the outburst is a Eddington limited type I X-ray burst.

\section{IGR J17391-3021/XTE J1739-302}

\subsection{Archival X-ray observations of the source}

The fast transient source IGR J17391-3021 was discovered in outburst with the IBIS/ISGRI detector on 26 August 2003 at
18:49 UT (Sunyaev et al. 2003) with a flux level of $70 \mathrm{mCrab}$ in the energy range $18-50 \mathrm{keV}$. The maximum flux (150 mCrab) was detected on 27 August 2003 at 00:44 UT, when the source was also detected in the hard energy range 50-100 keV with a flux of $50 \mathrm{mCrab}$. The total length of the outburst was less than a day. The 20-100 keV spectrum of IGR J17391-3021 at the maximum of the outburst was well fitted by an optically thin thermal bremsstrahlung model having a temperature of $T \sim$ $22 \mathrm{keV}$ (Lutovinov et al. 2005). To date, this is the only outburst of IGR J17391-3021 detected by IBIS/ISGRI and reported in the literature.

The IGR source position reported by Sunyaev et al. (2003), which has an error circle radius equal to $3^{\prime}$, is located $\sim 1.5^{\prime}$ from the Chandra location of the known transient XTE J1739-302, which has a position accuracy better than a few arcsec (Smith et al. 2004), so it cannot be excluded that IGR J17391-3021 and XTE J1739-302 are the same source. The RXTE satellite pointed to XTE J1739-302 35 h after the time quoted as the beginning of the previous cited outburst of IGR J17391-3021 detected by ISGRI but no detection was reported (Smith et al. 2003).

XTE J1739-302 (Smith et al. 1998) was the brightest source in the Galactic center region while active at the time of the RXTE discovery (12 August 1997), with a flux of $3 \times$ $10^{-9} \mathrm{erg} \mathrm{cm}^{-2} \mathrm{~s}^{-1}$ in the $2-25 \mathrm{keV}$ band. The source was only observed by RXTE in that one day, it was not detectable 9 days earlier or 2, 8 and 16 days later. The RXTE spectrum during the bright state is well described by a thermal bremsstrahlung model with $k T \sim 12 \mathrm{keV}$, furthermore no statistically significant periodicities have been found from 0.01 to $1000 \mathrm{~Hz}$. Smith et al. (1998) tentatively identify XTE J1739-302 as a Be/NS system, however its outbursts (constrained by RXTE observations to be more than a few hours but less than a day) are much shorter than those typical of Be/NS binaries systems. Due to this unusual transient behavior, XTE J1739-302 could define a new class of high mass fast X-ray transients (Smith et al. 2004).

ASCA detected XTE J1739-302 on 11 March 1999 (Sakano et al. 2002), 1.5 years after the RXTE discovery. The light curve (2-10 keV) initially showed no flux, then suddenly the source flared up reaching the peak after about $4 \mathrm{~min}$, then dropped to zero flux level with the same timescale as the flarerise. Then the source flared up again, this second flare being characterized by an almost identical profile except for the peak flux which was about half of that of the first flare. The $2-10 \mathrm{keV}$ ASCA spectrum of XTE J1739-302, accumulated during the flaring period, is a very hard and absorbed power law with an index corresponding to a temperature over $100 \mathrm{keV}$ when a bremsstrahlung model is applied. Hence the ASCA spectrum is significantly different in shape and also the reported column density is lower than the one measured by RXTE in August 1997. ASCA timing analysis did not find any significant pulsations, which is consistent with the RXTE results by Smith et al. (1998).

Recently, a Chandra observation of XTE J1739-302 in quiescence provided a very accurate position at RA = $17^{\mathrm{h}} 39^{\mathrm{m}} 11.58^{\mathrm{s}} \mathrm{Dec}=-30^{\circ} 20^{\prime} 37.6^{\prime \prime}$ with an position accuracy better than few arcsec (Smith et al. 2004). This permited the identification of its optical counterpart which is a bright highly 
Table 4. Newly discovered outbursts of IGR J17391-3021.

\begin{tabular}{clcccc}
\hline \hline No. & Date & Burst start time (UTC) & Burst stop time (UTC) & Energy range & flux at the peak (20-30 keV) \\
\hline 1 & 22 March 2003 & $12: 12: 44$ & $14: 00: 44$ & $20-60 \mathrm{keV}$ & $254 \mathrm{mCrab}$ \\
2 & 9 March 2004 & $06: 49: 44$ & $07: 19: 45$ & $20-40 \mathrm{keV}$ & $150 \mathrm{mCrab}$ \\
3 & 9 March 2004 & $11: 31: 48$ & - & $20-60 \mathrm{keV}$ & $280 \mathrm{mCrab}$ \\
4 & 10 March 2004 & $01: 54: 35$ & $03: 11: 23$ & $20-60 \mathrm{keV}$ & $250 \mathrm{mCrab}$ \\
\hline
\end{tabular}
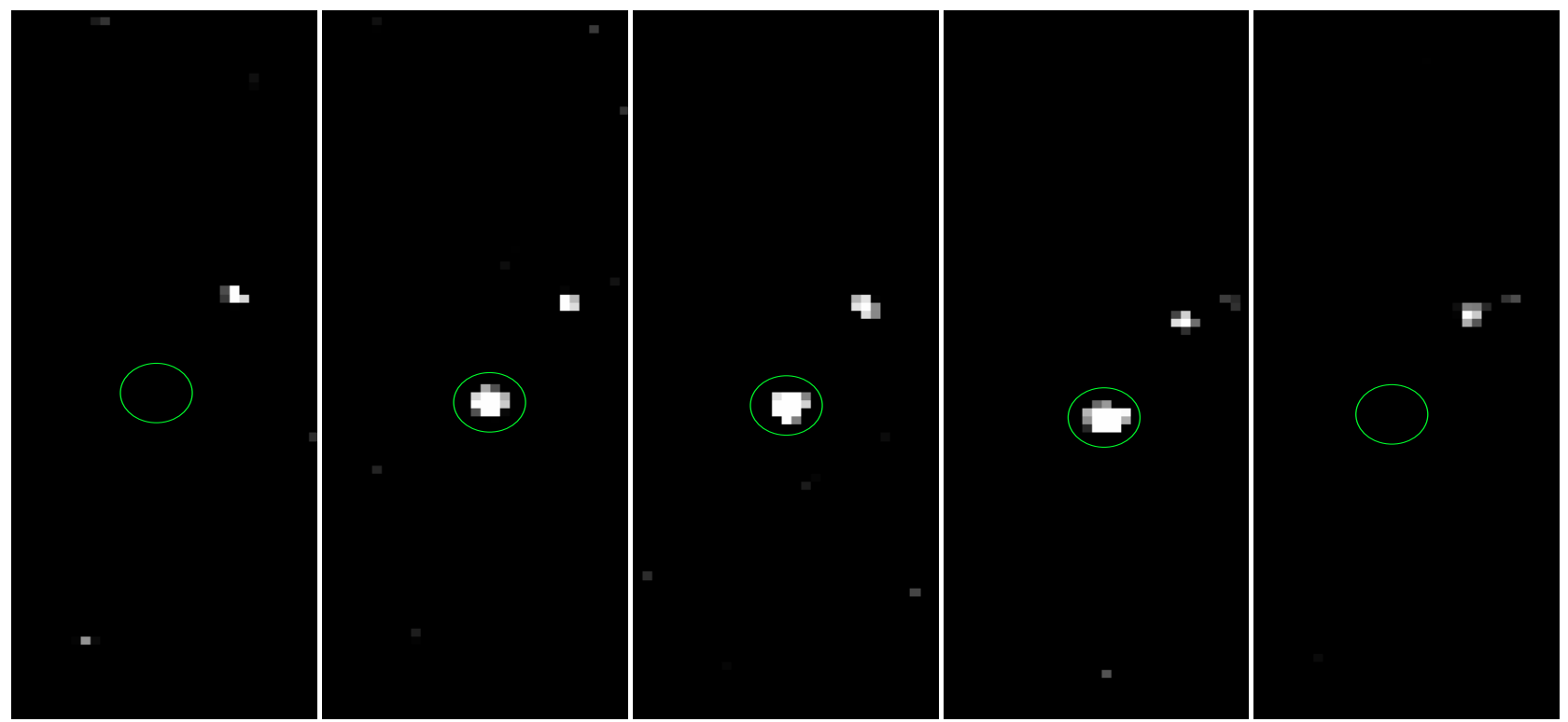

Fig. 8. ISGRI Science Window (ScW) image sequence (20-30 keV) of a newly discovered outburst (No. 1 in Table 4) of IGR J17391-3021/XTE J1739-302 (encircled). The duration of each ScW is $\sim 2000 \mathrm{~s}$. The source was not detected in the first ScW (significance less than $2 \sigma$ ), then it was detected during the next $3 \mathrm{ScWs}$ with a significance, from left to right, equal to $14 \sigma, 16 \sigma$ and $23 \sigma$, respectively. Finally in the last $\mathrm{ScW}$ the source was not detected (significance less than $2 \sigma$ ). A weak persistent source (1E 1740.7-2942) is also visible in the field of view.

reddened blue supergiant (O7.5-8Iaf) with no emission lines from an equatorial wind. Despite its position near the galactic center region, XTE J1739-302 could be a foreground object with a distance of about $1.8 \mathrm{kpc}$, as indicated by the bright optical companion.

\subsection{New results from analysis of IBIS/ISGRI observations}

We report on 4 newly discovered outbursts of IGR J17391-3021 from analysis of ISGRI data in revolutions No. 53 and No. 171. Firstly, our ISGRI analysis enabled a more accurate source position $\left(R A=17^{\mathrm{h}} 39^{\mathrm{m}} 08.52^{\mathrm{s}}\right.$ Dec $=-30^{\circ} 19^{\prime} 55.8^{\prime \prime}$, error circle radius $1.3^{\prime}$ ) than that reported by Sunyaev et al. (2003) when the source was discovered. Our improved position is now located $57^{\prime \prime}$ from the Chandra location of XTE J1739-302, this significantly increases the probability that the 4 newly discovered ISGRI outbursts and the one previously reported in the literature are from XTE J1739-302. The times of the 4 outbursts are listed in Table 4 together with the energy range over which they have been detected and their peak fluxes in the 20-30 keV band. We assumed the beginning of the first ScW during which the source was detected as being the start time of the outburst and similarly the burst stop time to be the end of the last $\mathrm{ScW}$ during which the source was visible. We note that their durations range from $\sim 30$ min to $\sim 2 \mathrm{~h}$, marking the very fast transient nature of IGR J17391-3021. The ISGRI light curves (20-30 keV) of the brightest outbursts (Nos. 1, 3 and 4 in Table 4) are shown in Figs. 9, 10 and 11, respectively.

Outburst No. 1 lasted $\sim 2 \mathrm{~h}$ and its ISGRI light curve (Fig. 9) shows three noticeable peaks. The first two peaks have a very fast rise and decay as well as a similar duration, both being $\sim 20 \mathrm{~min}$ long. The third peak lasts $\sim 1 \mathrm{~h}$ showing a fast rise followed by a slower decay. Furthermore, the last two flares have the same peak flux $(\sim 250 \mathrm{mCrab}$, energy band $20-30 \mathrm{keV})$ which is greater than that of the first flare $(\sim 170 \mathrm{mCrab}$, energy band $20-30 \mathrm{keV})$. Figure 8 shows the sequence of 3 consecutive ISGRI ScWs during which this outburst has been detected. It is worth noting that this outburst was detected by JEM-X. In the first and second ScW shown in Fig. 8, during which the outburst was detected by ISGRI, the source is first outside and then on the edge of the JEM-X field of view so it was not detected. On the contrary, during the last $\mathrm{ScW}$, the source is inside the FOV of JEM-X and it was detected, although at low significance $(9 \sigma, 9 \sigma, 7 \sigma$ and $4 \sigma$ respectively in the energy ranges $3-6,6-10,10-15$ and $15-35 \mathrm{keV})$. Unfortunately the statistics are not good enough to perform detailed spectral or timing analysis. 


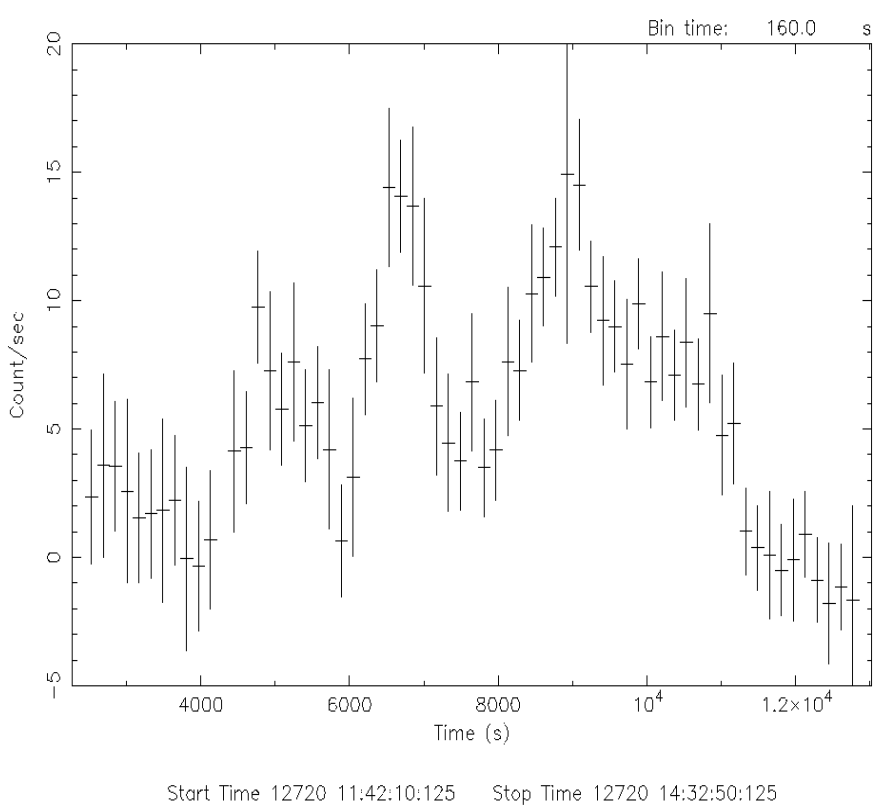

Fig. 9. ISGRI light curve (20-30 keV) of a newly discovered outburst of IGR J17391-3021 (No. 1 in Table 4).

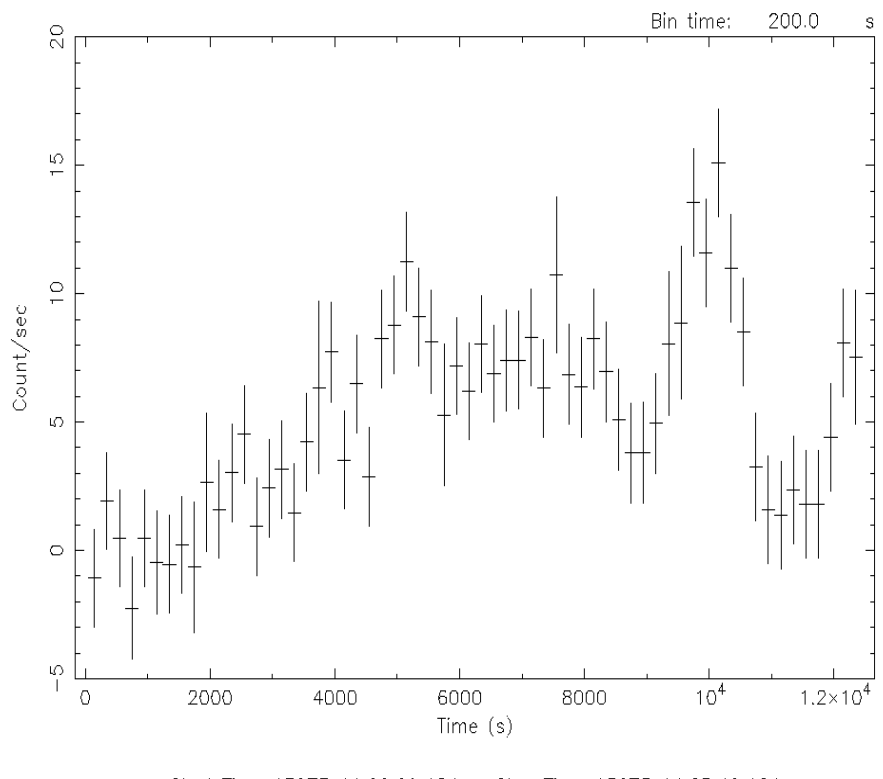

Fig. 10. ISGRI light curve (20-30 keV) of a newly discovered outburst of IGR J17391-3021 (No. 3 in Table 4).

Outburst No. 2 is the weakest and shortest one, being detected in only one $\mathrm{ScW}$ (providing an upper limit on its duration of $\sim 30 \mathrm{~min}$ ) up to $40 \mathrm{keV}$. Its peak flux is $150 \mathrm{mCrab}$ in the energy band 20-30 keV.

About five hours after outburst No. 2, the source turned on again (outburst No. 3 in Table 4). Its ISGRI light curve (Fig. 10) shows at the beginning a progressive rise lasting $\sim 80$ min during which the flux increases up to a value of $\sim 170 \mathrm{mCrab}$ between $20-30 \mathrm{keV}$. Then it stops increasing and for $\sim 50 \mathrm{~min}$ it does not show any drastic increase or decrease. The source subsequently flares up quickly reaching a peak flux value of $280 \mathrm{mCrab}$ and it drops quickly with the same timescale as

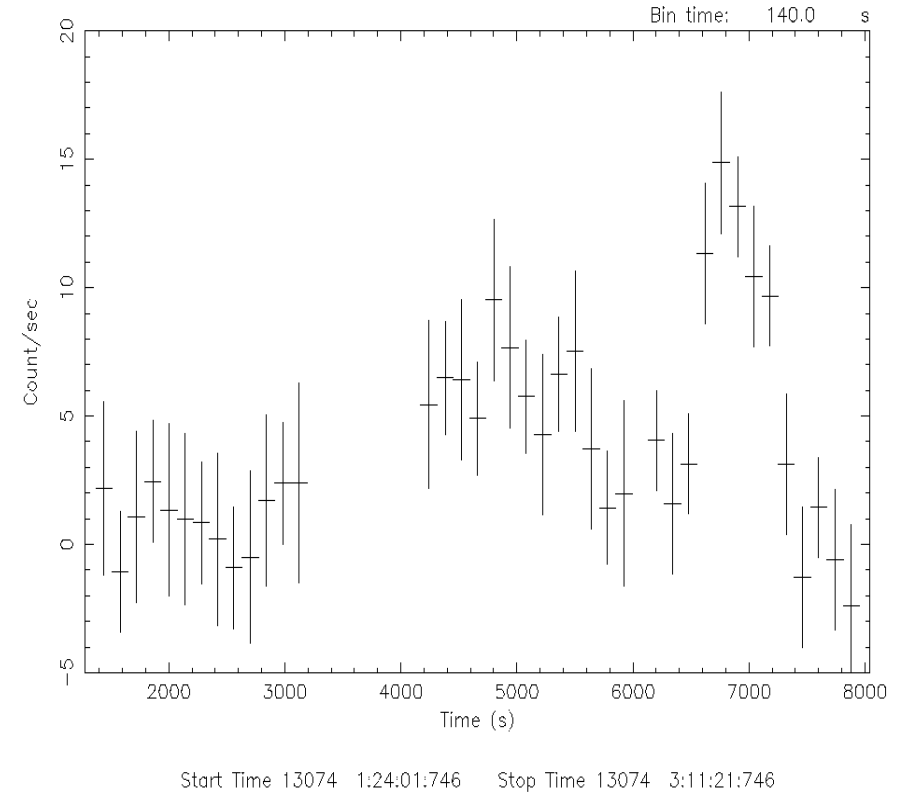

Fig. 11. ISGRI light curve (20-30 keV) of a newly discovered outburst of IGR J17391-3021 (No. 4 in Table 4).

the rise-time $(\sim 15 \mathrm{~min})$. There is evidence of a possible second peak but unfortunately the light curve is truncated at 14:27:12 UTC because the following $2 \mathrm{ScW}$ s are not available for data analysis. In this case it is not possible to establish exactly when the outburst terminates. However, we verified that the source was not detected in the next available $\mathrm{ScW}$ (starting at 15:24:01 UTC).

About 12 h after outburst No. 3, IGR J17391-3021 turned on again (outburst No. 4 in Table 4). In the light curve (see Fig. 11) there is a gap due to one unavailable $\mathrm{ScW}$ for data analysis, however the timing behavior of the source seems to suggest that it turned on just during the missing $\mathrm{ScW}$, so it is reasonable to assume the outburst start time at about 01:54:35 UTC. Once again, as seen in the previous outbursts, IGR J17391-3021 shows a very quick flare with a peak flux equal to $250 \mathrm{mCrab}(20-30 \mathrm{keV})$, then it drops to an undetectable flux level with the same timescale as the time-rise $(\sim 15 \mathrm{~min})$.

A Lomb-scargle periodogram analysis was performed to search for periodicities using the ISGRI data available from the four outbursts reported in Table 4, but no statistically significant periodicity has been found.

\section{Conclusions}

We have presented an analysis of IBIS/ISGRI data on newly discovered outbursts of three fast X-ray transient sources. The results confirm and strengthen the very fast transient nature of these sources by verifying that all the newly detected outbursts last less than a few hours. So far, FXTs did not show recurrence while all three fast transient sources here reported were detected in outburst by ISGRI more than once during the last 2 years, indicating for the first time a possible recurrent fast transient behaviour. 
In the case of the fast X-ray transient source SAX J1818.6-1703, we reported the detection with ISGRI of two outbursts that occured only $\sim 1.5$ days from each other. Their durations (respectively $\sim 1 \mathrm{~h}$ and $\sim 3 \mathrm{~h}$ ) are quite similar to that of the outburst detected by BeppoSAX WFCs when the source was discovered in $1998(\sim 2 \mathrm{~h})$.

In the case of IGR J16479-4514, there might be reasons to consider it a HMXB, based on its spectral characteristics and its apparent location in a star formation region (Norma region). However, the duration of the 4 newly discovered outbursts detected by ISGRI and reported in this paper last less than $3 \mathrm{~h}$, making them significantly shorter than the typical outbursts of HMXBs or Be/NS binaries. One of the four outbursts is particularly interesting, its ISGRI light curve (20-30 keV) shows a flare lasting $\sim 30 \mathrm{~min}$ and reaching a peak flux of $850 \mathrm{mCrab}$ (luminosity of $1.3 \times 10^{37} \mathrm{erg} \mathrm{s}^{-1}$, assuming the source is located in the Norma region). It is characterized by a rapid rise of $\sim 5 \mathrm{~min}$ followed by a slower exponential decay of $\sim 25 \mathrm{~min}$. This timing behavior could be typical of a thermonuclear type I X-ray burst, identifing IGR J16479-4514 as a LMXB containing a neutron star. The spectrum of the outburst (20-60 keV) is fitted by a black body with a temperature equal to $\sim 6 \mathrm{keV}$, which is greater than that typical of type I X-ray bursts (2-3 keV), although black body temperatures in excess of $3 \mathrm{keV}$ were observed for several sources (Lewin et al. 1993). Moreover the statistics are not good enough to find evidence of the cooling of the characteristic temperature of the X-ray spectrum. Hence this kind of explanation for the enhanced flux event of IGR J16479-4514 is inconclusive if only based on time behavior of the outburst.

XTE J1739-302 is an unusual transient source. First of all, our ISGRI analysis enabled a more accurate IGR J17391-302 position than that reported by Sunyaev et al. (2003) when the source was discovered. Our improved position is located only 57 arcsec from the Chandra location of XTE J1739-302, this significantly increases the probability that IGR J17391-302 and XTE J1739-302 are the same source. The spectral characteristics of XTE J1739-302 are typical of a neutron star binary, probably a HMXB given that its optical counterpart was identitifed with a blue supergiant. However, the duration of its outbursts are much shorter than those typical of HMXBs or Be/NS binaries systems, as confirmed by our reported ISGRI outburst detections with durations between $30 \mathrm{~min}$ and 3 h. Although their ISGRI light curves show different timing behavior, they are all characterized by one or more quick flares having similar short duration ( 15-20 min). An ASCA observation of XTE J1739-302 in March 1999 provided a light curve $(2-10 \mathrm{keV})$ showing the same kind of rapid flares. XTE J1739-302 could define a new class of high mass fast $\mathrm{X}$-ray transients with blue supergiant secondaries (Negueruela et al. 2005), their fast outbursts could be perhaps due to some kind of short ejection intrinsic to the secondaries. Another plausible explanation could be short viscous timescale in small accretion disks associated with wind accretion (Smith et al. 2004).

The unusual short and luminous outbursts of the three fast transient sources here reported make them particularly interesting. This kind of sources are difficult to detect due to their very short outbursts, so it seems plausible that many such systems wait to be discovered in the Galaxy. Ongoing observations of IBIS/ISGRI may yield further detections of such sources.

Acknowledgements. V. Sguera is grateful to R. Cornelisse and A. Tarana for useful discussions and suggestions and to J. L. Galache for providing the period analysis software. This research made use of data obtained from the HEASARC, SIMBAD and NED database. This research has been supported by University of Southampton School of Physics and Astronomy. AB, PU and AM thank ASI financial support via contract I/R/046/0.

\section{References}

Ambruster, C. W., Wood, K. S., Meekins, J. F., et al. 1983, ApJ, 269, 779

Bailey, J., Sparks, W. B., Hough, J. H., \& Axon, D. J. 1986, Nature, 322,150

Burns, J. O., Feigelson, E. D., \& Schreier, E. J. 1983, ApJ, 273, 128

Catanese, M., Bradbury, S. M., Breslin, A. C., et al. 1997, ApJ, 487, L143

Cornelisse, R., \& Kuulkers, E. 2002, A\&A, 382, 174

Cornelisse, R., Heise, J., Kuulkers, E., et al. 2000, A\&A, 357, L21

Courvoisier, T. J. L., Walter, R., \& Beckmann, V. 2003, A\&A, 411, L53

Dickey, \& Lockman 1990, ARA\&A, 28, 215

Ebisuzaki, T., \& nomoto, K., ApJ, 305, L67

Favata, F. 1998, Nucl. Phys. B, 69/1-3,23

Foster, A. J., Ross, R. R., \& Fabian, A. C. 1986, MNRAS, 221, 409

Garcia, M., Baliunas, S. L., Conroy, M., et al. 1980, ApJ, 240, L107

Heise, B. M., Brinkman, A. C., Schrijver, J., et al. 1975, ApJ, 202, L73

Heise, B. M., J. in 't Zand, J., Kippen, M., et al. 2001, in Gamma-Ray Bursts in the Afterglow Era, ed. E. Costa, F. Frontera, \& J. Hjorth (Berlin Heidelberg: Springer), 16

Hoffman, J. A., Lewin, W. H. G., Doty, J., et al. 1978, ApJ, 221, L57

in't Zand, J., Heise J., Smith, M., Muller, J. M. 1998, IAUC, 6840

Kuulkers, E. 2001, ATEL, 68

Kuulkers, E., in't Zand, J., van Kerkwijk, M. H., et al. 2002, A\&A, 382,503

Lebrun F., Leray, J. P., Lavocat, P., et al. 2003, A\&A, 411, L141

Lewin, W. H. G., \& Joss, P. C. 1981, Space Sci. Rev., 28, 3

Lewin, W. H. G., \& Vacca, W. D. 1984, ApJ, 277, L57

Lewin, W. H. G., van Paradijs, J., \& Taam, R. E. 1993, Space Sci. Rev., 62, 223

London R. A., Taam, R. E., \& Howard, W. M. 1984, ApJ, 287, L27

Lund, N., Butz-Jorgentsen, C., Westergaard, N. J., et al. 2003, A\&A, 411, L231

Lutovinov, A., Revnivtsev, M., Gilfanov, M., et al. 2004, [arXiv: astro-ph/0411550]

Lutovinov, A., Revnivtsev, M., Molkov, S., \& Sunyaev, R. 2005, A\&A, 430, 997

Maraschi, L., Fossati, G., Tavecchio, F., et al. 1999, ApJ, 526, 181

Molkov, S., Mowlavi, N., Goldwurm, A., et al. 2003, ATEL, 176

McHardy, I. M., Pye, J. P., Fairall, A. P., et al. 1982, MNRAS, 201, $31 \mathrm{P}$

Negueruela, I., Smith, D. M., \& Chaty, S. 2005, ATEL, 429

Reich, W., Steppe, H., Schlickeiser, R., et al. 1993, A\&A, 273, 65

Revnivtsev, M., Sunyaev, R., Varshalovich, D., et al. 2004, A\&AL, 30, 382

Sakano, M., Koyama, K., Murakami, H., Maeda, Y., \& Yamauchi, S. 2002, ApJS, 138, 19

Schnopper H. W., Delvaille, J. P., Epstein, A., et al. 1976, ApJ, 210, L75 
Smith, D. M., Main, D., Marshall, F., et al. 1998, ApJ, 501, L181

Smith, D. M., Markwardt, C. B., Swank, J. H., \& Heindl, W. A. 2003, ATEL, 186

Smith, D. M., \& Heindl W. A. 2004, ATEL, 218

Smith, D. M., Negueruela, I., \& Heindle, W. A. 2004, Americal Astronomical Society, HEAD meeting 8.2502S

Smith, D. M. 2004, ATEL, 338

Stern, R. A., Riegler, G. R., Agrawal, P. C., et al. 1981, Nature, 290, 573

Strohmayer, T. E., \& Bildsten, L. 2003, in Compact Stellar X-Ray Sources, ed. W. H. G. Lewin, \& M. van der Klis (Cambridge University Press), in press [arXiv:astro-ph/0301544]

Strohmayer, T. E., \& Brown E. F. 2002, ApJ, 566, 1045
Sunyaev R., Lutovinov, A., Molkov, S., et al. 2003, ATEL, 181

Swank, J. H, Becker, R. H., Boldt, E. A., et al. 1977, ApJ, 212, L73

Tawara, Y., Kii, T., Hayakawa, S., et al. 1984a, ApJ, 276, L41

Tawara, Y., Hayakawa, S., Hayakawa, S., \& Kii, T. 1984b, PASJ, 36, 845

Ubertini, P., Lebrun, F., Di Cocco, G., et al. 2003, A\&A, 411, L131

Vadim A. A., William, C., \& Konstantin, N. 2003, ApJ, 586, 1238

Volk, K., \& Cohen, M. 1989, AJ, 98, 931

Wijnands, R. 2001, ApJ, 554, L59

Winkler, C., Courvoisier, T. J.-L., Di Cocco, G., et al. 2003, A\&A, 411, L1 